\title{
The effect of short-term strength intervention on muscle activity of shoulder girdle during simulated crawl in elite swimmers
}

\author{
Simona Kubová*, Dagmar Pavlů, David Pánek, Vladimír Hojka, Radim Jebavý, and Kryštof Kuba \\ Faculty of Physical Education and Sport, Charles University, Prague, Czech Republic
}

Copyright: (c) 2020 S. Kubová et al. This is an open access article licensed under the Creative Commons Attribution License (https://creativecommons.org/licenses/by/4.0/).

Background: Specific strength intervention can influence muscle activity during swimming performance and can increase pain. Objective: The aim of this study was to assess the effect of strength intervention on muscle activity and pain during simulated crawl in elite swimmers. Methods: Fifteen elite Czech swimmers (4 females, 11 males) within the age of $22.8 \pm 3.4$ years participated in this study. At the baseline, muscle activity and pain evaluation was performed. For the shoulder joint pain assessment, the visual numeric scale (VNS) and the Swimmer's Functional Pain Scale (SFPS) questionnaires were used. Participants underwent surface electromyography (SEMG) examination of 8 evaluated muscles (trunk and upper arm) during simulated a 100 metres crawl race on swimming ergometer (VASA SwimErg, Essex Junction, Vermont, USA). After a 30 minutes long strength intervention, 100 metres crawl SEMG, VNS and SFPS were evaluated again. The smallest important change in muscle activation was set to $10 \%$. Results: The effect of strength intervention on muscle activity was not significant in all muscles, however effect size evaluation showed small effect of intervention in biceps brachii $(p=.311, d=0.33)$, upper part of trapezius $(p=.033, d=0.46)$ and serratus anterior $(p=.103, d=0.22)$ and medium effect in lower part of trapezius $(p=.053, d=0.62)$. Magnitude-based decision analysis showed a highly increased muscle activity in biceps brachii. Also in serratus anterior muscle activity increased as well. Difference in shoulder joint pain before and after intervention of the dominant upper limb according to the SFPS questionnaire and VNS was not statistically significant. Conclusion: A 30 minutes long strength intervention had a significant effect on the muscle activity of the upper part of $\mathrm{m}$. trapezius. There was no significant effect of the intervention on pain.

Keywords: swimming, electromyography, strength intervention, shoulder joint, pain

\section{Introduction}

Crawl is the fastest swimming stroke and it is the most frequently used swimming stroke during training. The speed of the swimming locomotion is relatively smooth, with only slight fluctuations of speed during the swimming cycle. Crawl requires a slightly inclined head with a downward-looking eyesight that keeps the hips and the entire body horizontal with the surface (Maglischo, 2003; McLeod, 2010).

The basic muscles that are activated while swimming crawl include $\mathrm{m}$. deltoideus and $\mathrm{m}$. supraspinatus, which are activated primarily when entering and leaving the upper limb below the surface, $\mathrm{m}$. rhomboideus

\footnotetext{
* Address for correspondence: Simona Kubová, Department of Physiotherapy, Faculty of Physical Education and Sport, Charles University, José Martího 31, 16252 Prague 6, Czech Republic. E-mail: s.baumrtova@seznam.cz
}

major securing the position of the shoulder blade for the movement of the upper limb, m. latissimus dorsi used to maintain the position of the upper limb and the position of the body, $\mathrm{m}$. serratus anterior and $\mathrm{m}$. subscapularis working constantly during the swim and $\mathrm{m}$. infraspinatus that is providing the function of the external rotator of the upper limb during relaxation. The lower limbs muscles that accompany the upper limbs while swimming crawl are as follows mm. glutei, m. quadriceps femoris, ischiocrural muscles and $\mathrm{m}$. triceps surae (Maglischo, 2003; Martens, Figuiredo, \& Daly, 2015).

The repetition of movement cycles during long and demanding swimming training, which the swimmers are undergoing for several years, are becoming a major etiological factor for the emergence of the so-called "swimming shoulder" (Pink, Perry, Browne, Scovazzo, \& Kerrigan, 1991). Richardson, Jobe, and Collins (1980) found out that shoulder pain occurs in 
$52 \%$ of elite swimmers and $27 \%$ of non-elite swimmers. McMaster and Troup (1993) discovered shoulder pain in $47 \%$ of 10 - to 18 -year-old swimmers, $66 \%$ of top swimmers and $73 \%$ of elite swimmers. The frequency of pain in both studies was the highest among elite swimmers, which may correlate with increased training doses and training times (Heinlein \& Cosgarea, 2010). The occurrence of pain and injuries in the area of shoulder joints is often times associated with athletes with poor technique of movement cycle and muscle fatigue. The occurrence of trigger points in the muscles involved in swimming locomotion is enormous. Trigger points are a great source of pain (Kolár, 2009). Pain can be a common problem for crawlers on the side that they are not normally taking their breath because they use that side as support for breathing in (Pink et al., 1991). It can be stated that the shoulder pain is caused by insufficient warm-up before each training, incorrect technique of swimming locomotion, insufficient compensation and lack of regeneration (Batalha et al., 2020). The most common causes of the development of the swimming shoulder, that are reported by the studies, are the long-term overload of work on the shoulder joint (Couanis, Breidahl, \& Burnham, 2015; Lintner, Noonan, \& Kibler, 2008; Manske, Lewis, Wolf, \& Smith, 2015), sexual dimorphism and swimming specialization (Hawley, Myburgh, Noakes, \& Dennis, 2010; Wymore, Reeve, \& Chaput, 2012), anatomical abnormalities of the shoulder joint (Page, 2011; Struyf, Nijs, De Graeve, Mottram, \& Meeusen, 2011), increased muscle tension and tendinopathy of m. supraspinatus (Sein et al., 2010).

Top swimmers usually have several sessions throughout the day. Usually, swimmers complete between 8-10 swim training units per week for a total duration of 90 to 180 minutes per training unit. To do this, they take 3 to 6 strength units a week for a duration of 30 to 120 minutes per training unit. For this research, the most commonly used type of training in the Czech Republic was chosen and the strength intervention took place at a minimum rest (up to 15 minutes) from the simulated race on swimming ergometer VASA (Eider, 2014).

In a study written by Morouço et al. (2011), it was reported that 11 of the 23 elite swimmers examined had transient shoulder pain during and after exercise at a high resistance gym. Similar results were obtained by Martins, Paiva, Freitas, Miguel, and Maya (2014) and Stirn, Jarm, Kapus, and Strojnik (2011).

Surface electromyography (SEMG) is a diagnostic method used primarily to evaluate the level of muscle activity during various movements, as well as to evaluate the timing of muscles, which is of great importance in assessing the quality of the movements performed. Many studies used SEMG to clarify the problem of swimming technique, to find out the cause of the pain or help identify a new movement stereotype (Mills, 2005).

From a physiotherapeutic point of view, variation in muscle activity changes the movement stereotype and thus can cause pain (Koláŕ, 2009). Muscle imbalances frequently exist due to the biomechanics of the swimming, which predisposes swimmers to injury or pain (Hibberd, Oyama, Spang, Prentice, \& Myers, 2012). Several studies have reported the impact of strength training on increased muscle activity in the shoulder girdle area among swimmers (Batalha et al., 2015; Gatta et al., 2015). Many studies reported, that the different muscle activations in strength training can also occur when performing the same exercise with just a change in the tilt angle during exercise and may increase shoulder pain (Lauver, Cayot, \& Scheuermann, 2016; Trebs, Brandenburg, \& Pitney, 2010). The aim of this study was to assess the effect of strength intervention on muscle activity and pain during simulated crawl in elite swimmers.

\section{Methods}

\section{Sample}

The research group included all national swimming team members of the Czech Republic, who specialized in crawl as their main swimming stroke. There were 15 participants in the research including 11 males (age $21.7 \pm 3.2$ years, body weight $81.6 \pm 6.7 \mathrm{~kg}$, body height $186.9 \pm 5.0 \mathrm{~cm}$, training period $11.64 \pm 1.6$ years) and 4 females (age $25.5 \pm 2.0$ years, body weight $60.5 \pm 3.2 \mathrm{~kg}$, body height $172 \pm 3.7 \mathrm{~cm}$, training period $13.25 \pm 0.4$ years). The experiment was approved by the Ethics Committee of Faculty of Physical Education and Sport of Charles University under reference number 86/2016. Each participant signed informed consent.

\footnotetext{
Measures

Approximately 60 minutes were set aside for each participant and all examinations were performed within one day. For each selected participant who participated in this study, data on the incidence, onset and location of pain in the shoulder joint were carefully recorded using the visual numeric pain scale (VNS) and the Swimmer's Functional Pain Scale (SFPS) questionnaire. Prior to the start of the research, it was necessary to submit a confirmation by a specialist that ultrasound examination was performed in the area of the shoulder girdle of all participants in order to exclude structural damage to the examined area. The cervical spine was examined by a specialist using the physiotherapeutic method Mechanical Diagnostic and Treatment with no
} 
pathological findings and with no clinical connection to shoulder pain. Subsequently, the participant was asked for the second part of the examination, which took place in the laboratory and gym at Faculty of Physical Education and Sport of Charles University in Prague.

For the SEMG examination, telemetry 16-channel EMG TelemyoMini 16 (Noraxon, Scottsdale, AZ, USA) was used. The examination was performed using special bipolar electrodes made of $\mathrm{Ag} / \mathrm{AgCl}$ material with a diameter of $5 \mathrm{~mm}$ with a set of double-sided adhesive labels necessary for firm adhesion of the electrodes to the skin (Mills, 2005). Before applying the surface electrodes, it was necessary to provide means for cleaning the electrode sticking area by shaving the hair, coarse cleaning with abrasive paper, and degreasing the area with cellulose squares and attaching EMG cables to the body with Leukopor tape. Electrode placement was chosen according to anatomically valid standards and the electrodes were placed on the dominant upper limb and adjacent trunk (Figure 1).

The muscle activity of the following eight muscles was measured: $\mathrm{m}$. deltoideus pars anterior, m. trapezius upper and lower parts, which are activated mainly when entering and leaving the upper limb above the surface, m. serratus anterior, m. biceps brachii capitis longus and $\mathrm{m}$. pectoralis major operating constantly during the engagement phase, $\mathrm{m}$. infraspinatus providing the function of the external rotator of the upper limb during relaxation, the $\mathrm{m}$. triceps brachii medial part ensuring completion of the engagement phase below the surface. By using the surface electrodes, it was possible to compare the value of muscle activity to the maximum voluntary contraction.

\section{Procedures}

All selected participants marked any number they considered to be the current expression of the pain at rest

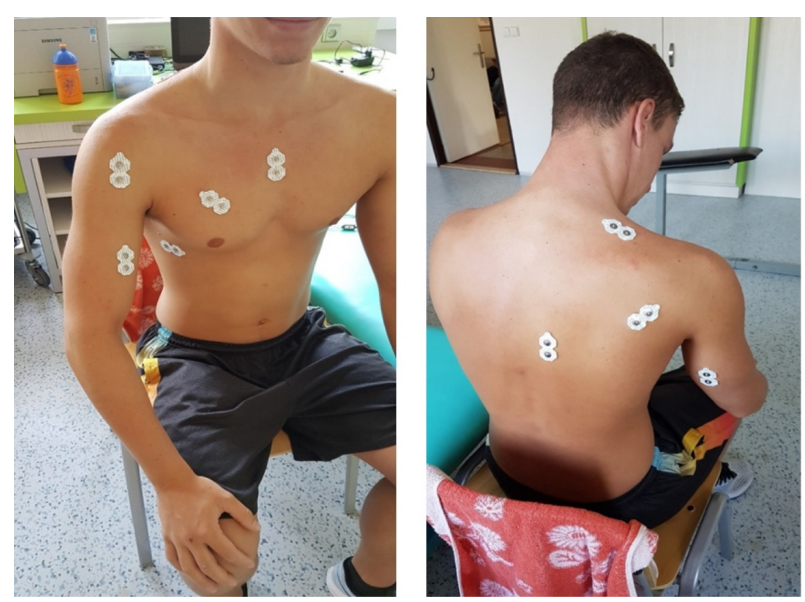

Figure 1. Placement of surface electrodes. and at work, on a numerical pain scale of $0-10$, with 0 as "no pain" and 10 as "severe pain" (Ritter, González, Laurent, \& Lorig, 2006). The participants also chose one number from 0 to 10 from the SFPS questionnaire survey as specific evaluation of shoulder pain in relation to swimming, where 0 was "no pain during swimming, either at practice and competitive event" and 10 was "the shoulder pain lasted more than 7 consecutive days" (Drake et al., 2015).

The maximum voluntary contraction was performed for 5 seconds of maximum possible strength according to the position of the muscle test (Janda, 2004) followed by a two-minute rest, and the entire test was performed three times for each muscle examined. The SEMG examination was then carried out on the swimming ergometer (VASA SwimErg, Essex Junction, VT, USA) and the muscle activity of the above muscles was detected by swimming at 70 cycles per minute for 60 seconds without inhalation because based on the results of the study Vezos et al. (2007) was revealed that the breath performed during the swimming sprint significantly prolonged the total duration of the movement cycle and there was a variation in the motor pattern, which was changed due to the rotation of the body during the breath.

After the SEMG examination, the electrodes remained attached to the participant bodies. The electrodes were attached with adhesive tape to prevent them from peeling off. Each participant underwent a 30-minute strength training session in the gym under the guidance of a fitness specialist. Following the strength intervention, the same SEMG examination was performed as before the intervention; the results of both examinations were compared, i.e., the muscle activity of all examined muscles was compared with the data from the initial measurement. SEMG examination was conducted under the guidance of a specialist (Kotalíková, Pánek, \& Pavlů, 2015).

\section{Intervention}

The strength intervention lasted for 30 minutes. It was applied in the mode of strength endurance with the magnitude of the load up to $50 \%$ of maximum in the main strength exercises focused on large muscle groups in the specified medium pace and the number of repetitions executed in medium tempo combined with explosive strength. This was followed by a medium-speed circuit practice focused on the endurance strength of the upper limbs and torso. Complementary exercises used only low resistances up to $5 \%$ of the maximum. Strength training was preceded by 5 minutes of warmup. Strength training was assembled on the basis of literary sources, internships and several practices with 
top swimmers and is described below (Berryman et al., 2018; Gatta et al., 2015; Jebavý, 2012).

\section{Endurance strength and casts}

Six repeititions of pitch (40-50\% of maximum) + explosive medicine ball throwing into the ground 10 repetitions, rest 30 seconds, 5 rounds, medium pace, aim for maximum speed as much as possible.

\section{Double circuit training}

Two rounds, rest 15 seconds, after two exercises rest 30 seconds, after the first round rest 1 minute, medium pace:

- maximum repetitions of pull-ups (it was possible to use a rubber band for the last 1-2 repetitions),

- body rotation with one end of the axis in the upper limb and the other end on the ground: 10-6 repetitions on both sides,

- dumbbells push-ups with pull-ins to the body: $10-8$ times with regular exchange,

- forward bend: 12-8 repetitions with forehead supported,

- rotation with $5 \mathrm{~kg}$ disc around the head: $10-8$ repetitions on both sides (change direction only after all repetitions have been completed),

- alternate leg raises in laying down position: 10-8 repetitions for both legs.

After the strength intervention, SEMG measurement followed without stretching in the time interval of 10 minutes (after the walk to the laboratory).

\section{Data processing}

The evaluation and analysis of the obtained data were analysed using the program MyoResearch (Version 3.14 XP Master Edition; Noraxon, Scottsdale, AZ, USA) with simultaneous video recording. When evaluating the SEMG signal it was necessary to remove movement and electrocardiogram artifacts, the signal was rectified and smoothed in the MyoResearch program (Kotalíková et al., 2015). When processing SEMG records of swimming locomotion, 10 movement cycles (10 right and 10 left of the upper limb) were selected, so as not to change the movement pattern, even if the electrodes were placed only on the dominant upper limb. The average amplitude was expressed in the relation to the percentage of maximum voluntary contraction of the examined muscle. For statistical data processing, basic descriptive statistics were first performed, where the averages of muscle activation values for each measured movement and their standard deviation were calculated.

Shapiro-Wilk test that is usually applied to small samples confirmed normal data distribution of muscle activity amplitudes. Muscle activity before and after intervention was compared by paired $t$-test. A $p$ value $<.05$ was considered significant. Effect size was evaluated using Cohen's $d$. According to Cohen (1992), the effect is described as small 0.2, medium 0.5 and large 0.8 .

Magnitude-based decision was used to evaluate the clinical significance of intervetion. It helps to understand the relation of confidence intervals and the smallest significant effect (beneficial, trivial, or harmful) (Hooren, 2018; Hopkins, 2019). The smallest significant effect in muscle activation was set at $10 \%$ (Kolář, 2009).

VNS was used to assess pain, which is one of the most commonly used pain measurement method (Kolář, 2009; Ritter, González, Laurent, \& Lorig, 2006). SFPS focuses on pain in swimming functional activities. For the purposes of this research, a translation into the Czech language of the standardized SFPS questionnaire was prepared (Drake et al., 2015; Pink et al., 1991). Differences in VNS and SFPS before and after intervention were evaluated by the Wilcoxon signed-rank test (Marino, 2018).

\section{Results}

Strength intervention in the total duration of $30 \mathrm{~min}$ utes had no effect on the change in muscle activity in muscles of the $\mathrm{m}$. pectoralis major, $\mathrm{m}$. triceps brachii, $\mathrm{m}$. infraspinatus and $\mathrm{m}$. deltoideus anterior in the highintensity swimming of elite swimmers. In $\mathrm{m}$. biceps brachii, the upper part of $\mathrm{m}$. trapezius and $\mathrm{m}$. serratus anterior, operating constantly during the engagement phase, the effect size was found small $0.33(p=.311)$, $0.46(p=.033)$, respectively $0.22(p=.103)$ after a 30-minute strength intervention for top swimmers. The highest increase in muscle activity after a thirty-minute strength intervention was found in the lower part of the $\mathrm{m}$. trapezius. The effect of the intervention in this muscle was medium $0.62(p=.053)$. Results are available in Table 1.

Statistical analysis of the results according to Hopkins (2019), magnitude-based decision, has shown a high increased muscle activity in $\mathrm{m}$. biceps brachii after the intervention program. Also in $\mathrm{m}$. serratus anterior and $\mathrm{m}$. pectoralis major the muscle activity increased. In the muscles of the trapezius upper and lower part and triceps brachii, magnitude-based decision has shown unclear effect. In the $\mathrm{m}$. deltoideus and $\mathrm{m}$. infraspinatus muscle activity after intervention decreased, the chance that the effect is beneficial was $82 \%$ and $88 \%$ respectively. Results of magnitude-based decision are presented in Table 2. 
Table 1

Comparison of muscle activity during $100 \mathrm{~m}$ crawl on the ergometer before and after strength intervention

\begin{tabular}{lccccc}
\hline & \multicolumn{2}{c}{ Muscle activity (\%MVC) } & & & \\
\cline { 2 - 3 } Muscle & Before & After & & Cohen's $d$ & \\
\hline Pectoralis major & $39.5 \pm 27.12$ & $39.6 \pm 30.91$ & & 0.00 & .990 \\
Deltoideus anterior & $11.3 \pm 11.02$ & $10.2 \pm 6.39$ & -0.13 & .594 \\
Biceps brachii & $7.7 \pm 3.55$ & $10.4 \pm 10.96$ & 0.33 & .311 \\
Triceps brachii & $48.4 \pm 19.66$ & $51.0 \pm 21.30$ & 0.13 & .237 \\
Upper part of trapezius & $4.9 \pm 6.22$ & $18.4 \pm 8.56$ & 0.46 & .033 \\
Infraspinatus & $17.7 \pm 8.50$ & $16.6 \pm 5.90$ & -0.15 & .532 \\
Lower part of trapezius & $28.7 \pm 7.24$ & $34.3 \pm 9.70$ & 0.62 & .053 \\
Serratus anterior & $14.6 \pm 8.73$ & $16.8 \pm 11.06$ & 0.22 & .103 \\
\hline
\end{tabular}

Note. $\quad \mathrm{MVC}=$ maximum voluntary contraction.

Table 2

Magnitude-based decision - the effect of intervention

\begin{tabular}{lcccl}
\hline & \multicolumn{3}{c}{ Chance (in \%) that true effect is } & \\
\cline { 2 - 4 } Muscle & Beneficial & Trivial & Harmful & Clinical decision \\
\hline Pectoralis major & 79 & 4 & 17 & Unclear - more data required \\
Deltoideus anterior & 3 & 14 & 82 & Likely < \\
Biceps brachii & 99 & 1 & 0 & Very likely > \\
Triceps brachii & 35 & 6 & 59 & Unclear - more data required \\
Upper part of trapezius & 49 & 44 & 7 & Unclear - more data required \\
Infraspinatus & 2 & 10 & 88 & Likely < \\
Lower part of trapezius & 14 & 17 & 69 & Unclear - more data required \\
Serratus anterior & 70 & 25 & 5 & Possibly >
\end{tabular}

The results of the VNS pain assessment correspond to the results of the SFPS questionnaire (Table 3). There was no significant difference in VNS and SFPS before and after intervention $(p=.510$, respectively $p=.534)$.

\section{Discussion}

The aim of the research was to analyse the effect of strength intervention on muscle activity and pain of selected muscle groups while swimming crawl by top swimmers in the Czech Republic. For this purpose, all national team members of the Czech Republic in swimming, who specialize in crawl with the age of $22.8 \pm 3.4$ years, regardless of sex, were included in the experiment.

Increase of muscle activity in the experiment in top swimmers occurred after strength intervention in total duration of 30 minutes in the upper part of $\mathrm{m}$. trapezius with a small effect size of $d=0.46(p=.033)$, $\mathrm{m}$. biceps brachii $d=0.33(p=.311)$ and $\mathrm{m}$. serratus anterior $d=0.22(p=.103)$. The only significant difference was found in the upper part of the m. trapezius which is activated when entering and leaving the upper limb above the surface during swimming locomotion. From a biomechanical point of view, it is activated primarily with elevation of upper limbs and tends to shorten (Maglischo, 2003). During strength training, unless the goal of the upper part of $\mathrm{m}$. trapezius is to strengthen, it strengthens as an undesirable phenomenon and a false stereotype of lifting weights (Jebavý, 2012). M. biceps brachii was activated the most during the transitional and pulling phases of crawl locomotion (McLeod, 2010). Excessive strain on the insertion of the $\mathrm{m}$. biceps brachii can result in pain in the shoulder area with frequent projection in the shoulder blade area (Wanivenhaus, Fox, Chaudhury, \& Rodeo, 2012). M. serratus anterior is working constantly during the swim, therefore its activity is necessary. So increased muscle activity in $\mathrm{m}$. serratus anterior shows the proper performance of the swimming technique (Maglischo, 2003; McLeod, 2010; Scovazzo, Browne, Pink, Jobe, \& Kerrigan, 1991). 
Table 3

Comparison of pain level before and after strength intervention

\begin{tabular}{lccccc}
\hline & \multicolumn{2}{c}{ VNS } & & \multicolumn{2}{c}{ SFPS } \\
\cline { 2 - 3 } \cline { 5 - 6 } Participant & Before & After & & Before & After \\
\hline 1 & 4 & 6 & & 4 & 8 \\
2 & 3 & 3 & & 3 & 3 \\
3 & 2 & 3 & & 2 & 3 \\
4 & 7 & 7 & & 7 & 7 \\
5 & 7 & 7 & & 7 & 7 \\
6 & 7 & 7 & & 7 & 7 \\
7 & 0 & 3 & & 0 & 2 \\
8 & 4 & 4 & & 4 & 4 \\
9 & 2 & 2 & & 2 & 2 \\
10 & 4 & 5 & & 5 & 6 \\
11 & 0 & 0 & & 0 & 0 \\
12 & 7 & 7 & & 7 & 7 \\
13 & 0 & 0 & & 0 & 0 \\
14 & 1 & 2 & & 1 & 2 \\
15 & 1 & 5 & & 1 & 7 \\
Median & 3 & 4 & 3 & 4 \\
\hline
\end{tabular}

Note. $\quad$ VNS $=$ Visual numeric scale; SFPS $=$ Swimmer's Functional Pain Scale.

After the strength intervention, the muscle activity of the lower part of the $\mathrm{m}$. trapezius increased with a medium effect size of $d=0.62(p=.053)$. However, this result is desirable. The lower part of the $\mathrm{m}$. trapezius activates during crawl at the entry and exit of the upper limb above the surface serves as a stabilizer of the shoulder blade and reduces the risk of the so-called swimming shoulder (Fig, 2010; Maglischo, 2003; McLeod, 2010).

In a study by McLaine, Bird, Ginn, Hartley, and Fell (2019) shoulder extension strength, a functional test for swimmers, was associated with and predictive of the development of shoulder pain in male swimmers. Low shoulder extension strength may be a risk factor for the development of shoulder pain in swimmers, proposing a direction for injury prevention and future investigation. In the study Morouco et al. (2011) it was reported that 11 of the 23 elite swimmers examined had transient shoulder pain during and after exercise at a high resistance gym due to increased muscle activity mainly in the upper part of m. trapezius. Similar results were obtained by the authors of the studies Martins et al. (2014) and Stirn et al. (2011).

The difference in the degree of pain was not statistically significant after the strength intervention.

But the question is, why muscle activity was not significantly increased. There are many factors that may have influenced the insignificant results of the research. The study may have been affected by the higher average age of women, although the training period was similar to men. With higher age, the muscle structure changes, especially the size of type II muscle fibres is reduced (Porter, Vandervoort, \& Lexell, 1995). Long-term competitive swim training is associated with declining measures of shoulder girdle muscles properties and self-reported measures of pain and function (Dischler, Baumer, Finkelstein, Siegal, \& Bey, 2018).

Another factor could be the standard strength training for swimmers was not set up properly or was incorrectly applied immediately before the start of the swimming test. The time interval between the dry-land and the swimming unit is very important. It is also likely that with a longer interval, the pain in the shoulders would be lower because there would be a time for longer regeneration after the strength intervention (Hibberd et al., 2012).

Several studies describe the effect of dry-land practise on increased shoulder pain, which is completed with a minimum time interval from the swimming training. At the same time, it is very important to mention what type of dryland unit is being performed. Whether it is a strength training, maximum strength, endurance training, bodyweight training. Because each unit has a different effect on muscle activity relating to shoulder pain. In the experiment, we used strength training (Berryman et al., 2018; Gatta et al., 2015; Girold et al., 2012).

There are very few scientific studies that look at the effect of strength training on shoulder pain among swimmers. Several studies have reported the impact of strength training on increased muscle activity in the shoulder girdle area after the strength intervention, but in both studies, it was a longer-term experiment with more participants (Batalha et al., 2015; Gatta et al., 2015). Different muscle activations in strength training can also occur when performing the same exercise with just a change in the tilt angle and may increase shoulder pain (Lauver et al., 2016; Trebs et al., 2010). Therefore, it is very important to perform strength exercises with a perfect technique (Jebavý, 2012). In the experiment, all strength exercises were performed under the guidance of a specialist.

Another factor could have been the current level of the swimming performnce. Although certain definitions of training that should have taken place prior to the research were established, extracurricular activities were not taken into account in the research. The current degree of fatigue of individual participants may have been different. It could have been another commitment to doing this research (Eider, 2014). 


\section{Conclusions}

The results of the experiment have shown a significant difference in the muscle activity of the upper part of $\mathrm{m}$. trapezius and a difference on the verge of the significance of the lower part of $\mathrm{m}$. trapezius. After the intervention, there was no significant difference in the shoulder pain.

\section{Acknowledgments}

This research was conducted within the Programme for the Development of Fields of Study at Charles University, No. P39 Biological aspects of the investigation of human movement.

\section{Conflict of interest}

There were no conflicts of interest.

\section{References}

Batalha, N., Paixão, C., Silva, A. J., Costa, M. J., Mullen, J., \& Barbosa, T. M. (2020). The effectiveness of a dryland shoulder rotators strength training program in injury prevention in competitive swimmers. Journal of Human Kinetics, 71, 11-20.

Batalha, N., Raimundo, A., Tomas-Carus, P., Paulo, J., Simão, R., \& Silva, A. J. (2015). Does a land-based compensatory strength-training programme influences the rotator cuff balance of young competitive swimmers? European Journal of Sport Science, 15, 764-772.

Berryman, N., Mujika, I., Arvisais, D., Roubeix, M., Binet, C., \& Bosquet, L. (2018). Strength training for middle-and long-distance performance: A meta-analysis. International Journal of Sports Physiology and Performance, 13, 1-27.

Cohen, J. (1992). A power primer. Psychological Bulletin, 112, 155-159.

Couanis, G., Breidahl, W., \& Burnham, S. (2015). The relationship between subacromial bursa thickness on ultrasound and shoulder pain in open water endurance swimmers over time. Journal of Science and Medicine in Sport, 18, 337-377.

Dischler, J. D., Baumer, T. G., Finkelstein, E., Siegal, D. S., \& Bey, M. J. (2018). Association between years of competition and shoulder function in collegiate swimmers. Sports Health, 10, 113-118.

Drake, S. M., Krabak, B., Edelman, G. T., Pounders, E., Robinson, S., \& Wixson, B. (2015). Development and validation of a swimmer's functional pain scale. Journal of Swimming Research, 23, 1-12.

Eider, P. (2014). Selection in swimming training. Central European Journal of Sport Sciences and Medicine, 5, 65-75.

Fig, G. (2010). Why competitive swimmers need explosive power. Strength and Conditioning Journal, 32, 84-86.
Gatta, G., Leban, B., Paderi, M., Padulo, J., Migliaccio, G. M., \& Pau, M. (2015). The development of swimming power. Muscles, Ligaments and Tendons Journal, 4, 438-445.

Girold, S., Jalab, C., Bernard, O., Carette, P., Kemoun, G., \& Dugué, B. (2012). Dry-land strength training vs. electrical stimulation in sprint swimming performance. Journal of Strength and Conditioning Research, 26, 497-505.

Hawley, J. A., Myburgh, K. H., Noakes, T. D., \& Dennis, S. C. (2010). Training techniques to improve fatigue resistance and enhance endurance performance. Journal of Sports Sciences, 15, 325-333.

Heinlein, S. A., \& Cosgarea, A. J. (2010). Biomechanical considerations in the competitive swimmer's shoulder. Sports Health, 2, 519-525.

Hibberd, E. E., Oyama, S., Spang, J. T., Prentice, W., \& Myers, J. B. (2012). Effect of a 6-week strengthening program on shoulder and scapular-stabilizer strength and scapular kinematics in Division I collegiate swimmers. Journal of Sport Rehabilitation, 21, 253-265.

Hooren, B. (2018). Magnitude-based inference: What is it? How does it work and is it appropriate? Sport Performance and Science Report, 1, 1-2.

Hopkins, W. G. (2019). Compatibility intervals and magnitude-based decisions for standardized differences and changes in means. Sportscience, 23, 1-4.

Janda, V. (2004). Svalový funkční test [Muscle function tests] (2nd ed.). Prague, Czech Republic: Grada.

Jebavý, R. (2012). Silový trénink v České republice a ve světě. Hodnocení roku 2011 [Strength training in the Czech Republic and in the world. Evaluation of the year 2011]. Paper presented at the Seminar for Coaches. Nymburk, Czech Republic.

Koláŕ, P. (2009). Rehabilitace v klinické praxi [Rehabilitation in clinical practice]. Prague, Czech Republic: Galén.

Kotalíková, K., Pánek, D., \& Pavlů, D. (2015). Kazuistika pacienta s Parkinsonovou nemocí - hodnocení chůze na suchu a ve vodě [Case study of patients with Parkinson's disease - electromyographic analysis of walking in various environment: On dry land and in water]. Rehabilitace a fyzikální lékařství, 22, 89-94.

Lauver, J. D., Cayot, T. E., \& Scheuermann, B. W. (2016). Influence of bench angle on upper extremity muscular activation during bench press exercise. European Journal of Sport Science, 16, 309-316.

Lintner, D., Noonan, T. J., \& Kibler, W. B. (2008). Injury patterns and biomechanics of the athlete's shoulder. Clinics in Sports Medicine, 27, 527-551.

Maglischo, E. W. (2003). Swimming fastest (2nd ed.). Champaign, IL: Human Kinetics.

Manske, R. C., Lewis, S., Wolf, S., \& Smith, B. (2015). Effects of a dry-land strengthening program in competitive adolescent swimmers. International Journal of Sports Physical Therapy, 10, 858-867.

Marino, M. J. (2018). Statistical analysis in preclinical biomedical research. In M. Williams, M. J. Curtis, \& K. Mullane (Eds.), Research in the biomedical sciences (pp. 107-144). Cambridge, MA: Academic Press.

Martens, J., Figuiredo, P., \& Daly, D. (2015). Electromyography in the four competitive swimming strokes: 
A systematic review. Journal of Electromyography and Kinesiology, 25, 273-291.

Martins, L. C. X., Paiva, J. R., Freitas, A. C., Miguel, L. B., \& Maia, F. R. C. (2014). Prevalence of pain and associated factors in elite swimmers. Science \& Sports, 29(3), e11-e14.

McLaine, S. J., Bird, M. L., Ginn, K. A., Hartley, T., \& Fell, J. W. (2019). Shoulder extension strength: A potential risk factor for shoulder pain in young swimmers? Journal of Science and Medicine in Sport, 22, 516-520.

McLeod, I. (2010). Swimming anatomy (2nd ed.). Champaign, IL: Human Kinetics.

McMaster, W. C., \& Troup, J. (1993). A survey of interfering shoulder pain in United States competitive swimmers. American Journal of Sports Medicine, 21, 67-70.

Mills, K. R. (2005). The basics of electromyography. Journal of Neurology, Neurosurgery and Psychiatry, 76(Suppl. 2), ii32-ii35.

Morouço, P., Neiva, H., González-Badillo, J. J., Garrido, N., Marinho, D. A., \& Marques, M. C. (2011). Associations between dry land strength and power measurements with swimming performance in elite athletes: A pilot study. Journal of Human Kinetics, 29A, 105-112.

Page, P. (2011). Shoulder muscle imbalance and subacromial impingement syndrome in overhead athletes. International Journal of Sports Physical Therapy, 6, 51-58.

Pink, M., Perry, J., Browne, A., Scovazzo, M. L., \& Kerrigan, J. (1991). The normal shoulder during freestyle swimming. An electromyographic and cinematographic analysis of twelve muscles. American Journal of Sports Medicine, 19, 569-576.

Porter, M. M., Vandervoort, A. A., \& Lexell, J. (1995). Aging of human muscle: Structure, function and adaptability. Scandinavian Journal of Medicine \& Science in Sports, 5, 129-142.

Richardson, A. B., Jobe, F. W., \& Collins, H. R. (1980). The shoulder in competitive swimming. American Journal of Sports Medicine, 8, 159-163.
Ritter, P. L., González, V. M., Laurent, D. D., \& Lorig, K. R. (2006). Measurement of pain using the visual numeric scale. Journal of Rheumatology, 33, 574-580.

Scovazzo, M. L., Browne, A., Pink, M., Jobe, F. W., \& Kerrigan, J. (1991). The painful shoulder during freestyle swimming. An electromyographic cinematographic analysis of twelve muscles. American Journal of Sports Medicine, 19, 577-582.

Sein, M. L., Walton, J., Linklater, J., Appleyard, R., Kirkbride, B., Kuah, D., \& Murrell, G. A. (2010). Shoulder pain in elite swimmers: Primarily due to swim-volumeinduced supraspinatus tendinopathy. British Journal of Sports Medicine, 44, 105-113.

Stirn, I., Jarm, T., Kapus, V., \& Strojnik, V. (2011). Evaluation of muscle fatigue during 100-m front crawl. European Journal of Applied Physiology, 111, 101-113.

Struyf, F., Nijs, J., De Graeve, J., Mottram, S., \& Meeusen, R. (2011). Scapular positioning in overhead athletes with and without shoulder pain: A case-control study. Scandinavian Journal of Medicine \& Science in Sports, 21, 809-818.

Trebs, A. A., Brandenburg, J. P., \& Pitney, W. A. (2010). An electromyography analysis of 3 muscles surrounding the shoulder joint during the performance of a chest press exercise at several angles. Journal of Strength and Conditioning Research, 24, 1925-1930.

Vezos, N., Gourgoulis, V., Aggeloussis, N., Kasimatis, P., Christoforidis, C., \& Mavromatis, G. (2007). Underwater stroke kinematics during breathing and breath-holding front crawl swimming. Journal of Sports Science \& Medicine, 6, 58-62.

Wanivenhaus, F., Fox, A. J., Chaudhury, S., \& Rodeo, S. A. (2012). Epidemiology of injuries and prevention strategies in competitive swimmers. Sports Health, 4, 246-251.

Wymore, L., Reeve, R. E., \& Chaput, C. D. (2012). No correlation between stroke specialty and rate of shoulder pain in NCAA men swimmers. International Journal of Shoulder Surgery, 6, 71-75. 